\title{
Coronavirus-19 Pandemic: Mothers and Infants
}

Joseph R. Hageman, MD

Every time I think I have a final version of an editorial about the Coronavirus-19 (COViD-19) pandemic, another new paper pops up from the New England Journal of Medicine, JAMA, or JAMA Pediatrics! I have published 2 COViD-19 editorials in Pediatric Annals $(1,2)$, the second of which was just published as an update a couple of days ago. (2) I sensed that mothers and babies are definitely at risk for the acquisition of the COViD-19 virus. Still, in general, the literature has suggested that the vast majority of them were relatively spared from severe illness and death.

In order to provide the most up to date recommendations for the management of newborns and mothers, we have an excellent fellow column by Smith and Sharma in this issue. (3) In addition, I have a few thoughts to add in this pearl about aspects of COViD-19 infection in mothers and newborns.

\section{"In order to provide the most up to date recommendations for the management of newborns and mothers, we have an excellent fellow column by Smith and Sharma in this issue. (3) In addition, I have a few thoughts to add in this pearl about aspects of COViD-19 infection in mothers and newborns."}

Let's talk about pregnant mothers and their infants. On the positive side, there is a new article by lqbal and colleagues just published in the New England Journal of Medicine about "an uncomplicated delivery in a patient with COViD-19 in the United States" in which a pregnant woman presented at 39 weeks' gestation with clinical symptoms and signs of COViD-19 with a positive PCR and was delivered on hospital day 3. (4) The infant had Apgar scores of 8 and 9 at one of five minutes of age and had no evidence of clinical COViD-19 infection. The neonate was separated from the mother and nourished with formula and expressed breast milk. Mother and baby were discharged on day 6 , with the mother feeling better. (3) Care was taken from the time of admission through discharge to keep from infecting hospital staff. The father also tested positive for COViD-19 (4). The follow up was uneventful for the infant, mother, and father. (4)

On the negative side, "maternal death due to COVID-19 disease" was published the day before by Hantoushzadeh, Shamshirsaz, and colleagues in a collaborative effort with clinicians in Iran and with Baylor College of Medicine and Texas Children's Hospital in the American Journal of Obstetrics and Gynecology(5). In this case series which summarizes the clinical courses of 9 pregnant women diagnosed with SARS-CoV-2 infection during their latter second and third trimester. All nine women were diagnosed with rRT-PCR nucleic acid testing (NAT): 7 of 9 died, 1 of 9 remains critically ill, and one recovered after a prolonged hospitalization (5). The authors provide an excellent summary of the current literature about maternal COViD-19 infection, which both reinforces the fact that the vast majority of pregnant women who acquire COViD-19 infection have a mild illness, but also that severe illness with acute respiratory distress syndrome is also possible. (5) The authors also report the level of illness of household contacts of these women, which was mild and the outcomes of their infants: five infants including a set of twins were intrauterine fetal deaths (IUFD), a set of 28-week gestation twins who died on day of life 3 from respiratory complications, one $305 / 7$ week premature infant who acquired COViD-19 infection on hospital day 3 , remains intubated in the neonatal intensive care unit, three other infants who are alive and doing well. (5) There was also a research letter by Baud and colleagues about a pregnant woman who had COViD-19 or SARS-CoV-2 infection and had a miscarriage in her second trimester. (6) The mother had a positive nasopharyngeal swab for COViD-19. The stillborn infant was delivered vaginally after ten hours of labor, and swabs from the axillae, mouth, meconium, and fetal blood were all negative. The fetal autopsy revealed no malformations, and fetal lung, liver, and thymus biopsies were also negative. Within minutes of expulsion, the fetal surface of the placenta was disinfected and incised, and two swabs and biopsies close to the umbilical cord and margin were obtained, all were negative for bacterial infection, but were positive for SARS-CoV-2. The placental histology showed mixed inflammatory infiltrates composed of neutrophils and monocytes in the subchorial space, and evidence of inflammation of the umbilical cord (funisitis) was also noted. (6) These findings suggest COViD-19 related placental infection. There was no evidence of vertical transmission of COViD-19; however, no other cause for the fetal demise was demonstrated. (6) The authors suggest that further investigation of whether SARS-CoV-2 crossed the placenta is warranted. (6)

\section{"The authors provide an excellent summary of the current literature about maternal COViD-19 infection, which both reinforces the fact that the vast majority of pregnant women who acquire COViD-19 infection have a mild illness, but also that severe illness with acute respiratory distress syndrome is also possible. (5)"}

The combination of the up to date summary by Smith and Sharma and these findings provide obstetrical providers, neonatologists, and pediatric providers' guidelines for the care of mothers with 
COViD-19 infection and their infants as well as their family/ household members.

\section{References}

1. Hageman JR. The Coronavirus disease 2019 (COViD-19). Pediatr Ann 2020;49(3):e99-e100.

2. Hageman JR. The evolving COVID-19 pandemic: An update. Pediatr Ann 2020;49(5):1-3.

3. Smith AG, Sharma AM. COVID-19 Clinical Quick Guide for the Neonatologist

4. Iqbal SN, Overcash R, Mokhtari et al. An uncomplicated delivery in a patient with Covid-19 in the United States. $N$ Engl J Med 2020; 382(16): e34.

5. Hantoushzadeh $S$, Shamshirsaz AA, Aleyasin $A$, et al. Maternal death due to COVID-19 disease. Am J Ob Gyne doi: htt[s://doi.org/10.1016/j.ajog.2020.04.030.

6. BaudD, Greub G, Favre G et al. Second trimestermiscarriage in a pregnant woman with SARS-COV-2 infection. JAMA 2020; published April 30, 2020; E1-E3.

The author has no conflicts to disclose

\section{NATIONAL PERINATALASSOCIATION}

\section{Update: CORONAVIRUS COVID-19}

According to data

published in The Lancet

Pregnant women with COVID-19 infection had fewer complications and adverse outcomes than would be anticipated for those with SARS-CoV-1 infection.

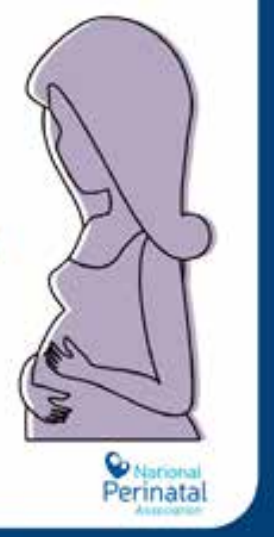

www.nationalperinatal.org

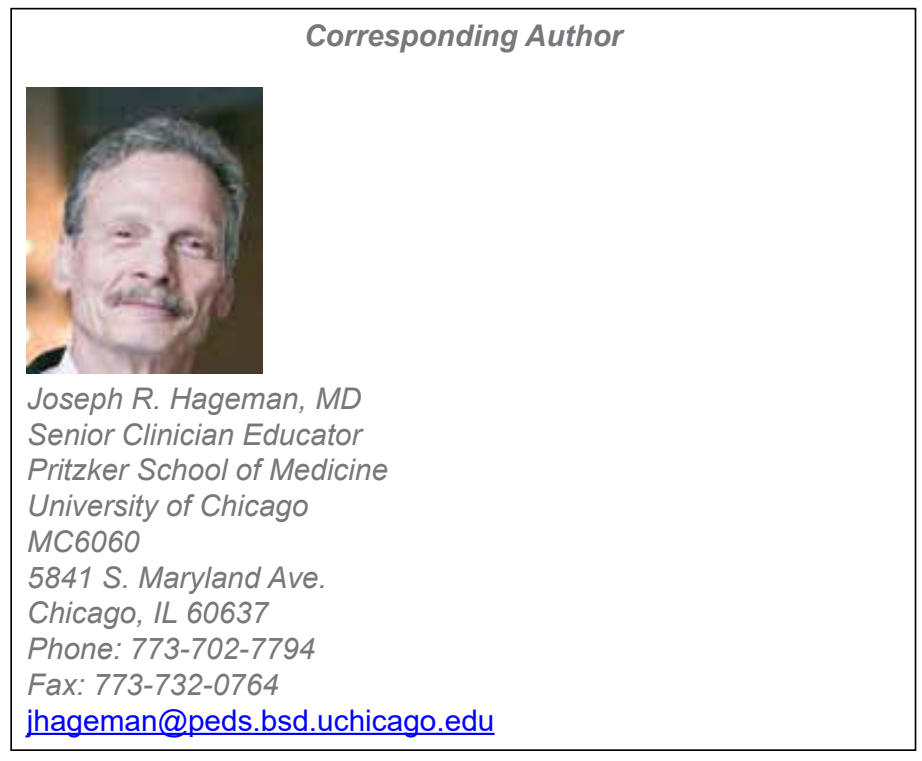

Clinical Pearls are published monthly.

Submission guidelines for "Clinical Pearls":

1250 word limit not including references or title page.

May begin with a brief case summary or example.

Summarize the pearl for emphasis.

No more than 7 references.

Please send your submissions to:

ihageman@peds.bsd.uchicago.edu

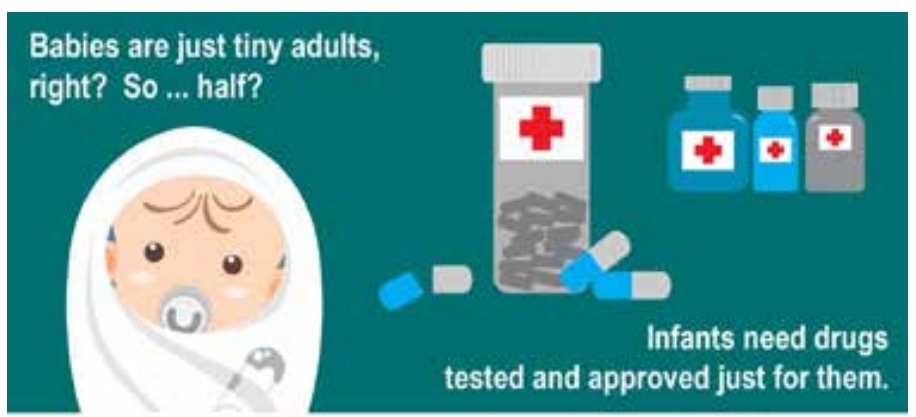

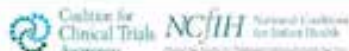

\section{THE \\ BRETT TASHMAN}

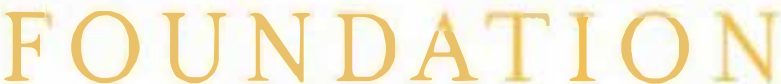

The Brett Tashman Foundation is a 501@(3) public charity. The mission of the Foundation is to find a cure for Desmoplastic Small Cell Round Tumors (DSRCT). DSRCT is an aggressive pediatric cancer for which there is no cure and no standard treatment. 100 percent of your gift will be used for research. There is no paid staff. To make your gift or for more information, go to "TheBrettTashmanFoundation.org" or phone (909) 981-1530. 